\title{
Nuclear Physics of Neutron Stars
}

\author{
J. Piekarewicz*1 \\ ${ }^{1}$ Department of Physics, Florida State University, Tallahassee, FL 32306
}

(Dated: September 3, 2021)

\begin{abstract}
Understanding the equation of state (EOS) of cold nuclear matter, namely, the relation between the pressure and energy density, is a central goal of nuclear physics that cuts across a variety of disciplines. Indeed, the limits of nuclear existence, the collision of heavy ions, the structure of neutron stars, and the dynamics of core-collapse supernova, all depend critically on the equation of state of hadronic matter. In this contribution I will concentrate on the special role that nuclear physics plays in constraining the EOS of cold baryonic matter and its impact on the properties of neutron stars.
\end{abstract}

PACS numbers: 97.60.Jd,26.60.+c,21.10.Gv

* e-mail: jpiekarewicz@fsu.edu 


\section{INTRODUCTION}

A neutron star is a gold mine for the study of the phase diagram of cold baryonic matter. Indeed, a remarkable fact about spherically symmetric neutron stars in hydrostatic equilibrium - the so-called Schwarzschild stars - is that the only physics that they are sensitive to is the equation of state of cold, neutron-rich matter. As such, neutron stars provide a myriad of observables that may be used to constrain poorly known aspects of the nuclear interaction under extreme conditions (both high and low) of density. Contrary to the most common perception of a neutron star as a uniform assembly of neutrons packed to densities that may exceed that of normal nuclei by up to an order of magnitude, the reality is far different and significantly more interesting. For example, the mere model-independent fact that hydrostatic equilibrium must be maintained throughout the star, generates a range of densities that span over 11 orders of magnitude; from about $10^{4}$ to $10^{15} \mathrm{~g} / \mathrm{cm}^{3}$ (nuclearmatter saturation density equals $\rho_{0}=2.48 \times 10^{14} \mathrm{~g} / \mathrm{cm}^{3}$ ). What novels phases of baryonic matter emerge under these extreme conditions is at present both fascinating and unknown. As we shall see, most of the exotic phases predicted to exist in neutron stars can not be realized under normal laboratory conditions. Whereas most of these phases have a fleeting existence in the laboratory, they become stable in neutron stars as a consequence of the presence of its enormous gravitational field. Understanding the physics underlying these phases will require a concerted and sustained effort from laboratory experiments, astronomical observations, and theoretical analyses. In this contribution I will aim to establish the particular role that nuclear physics plays in establishing some robust and enlightening correlations between the properties of finite nuclei and neutron stars.

\section{TOMOGRAPHY OF A NEUTRON STAR}

Neutron stars contain a non-uniform crust above a uniform liquid mantle that, in turn, is located above a possible exotic core. Figure 1 displays what is believed to be an accurate rendition of a neutron star (courtesy of Dany Page).

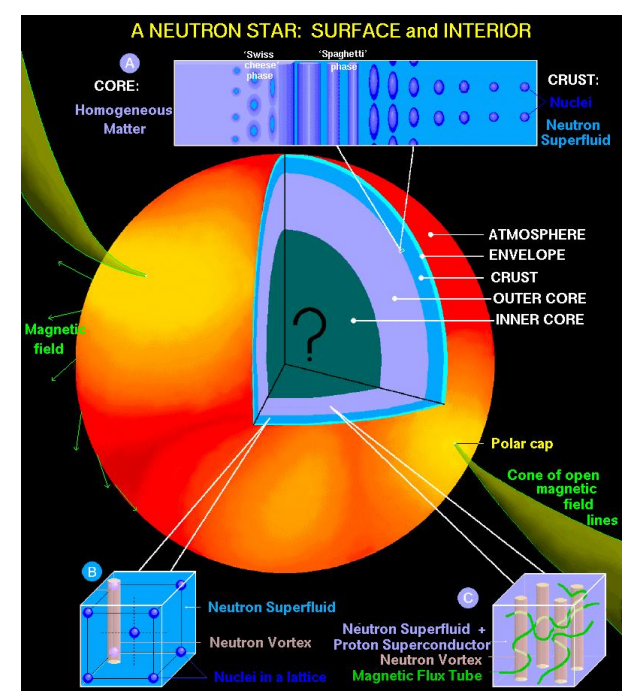

FIG. 1: A rendition of the structure and phases of a neutron star (courtesy of Dany Page). 


\section{A. The Outer Crust}

Neutron stars contain a non-uniform crust above the uniform liquid mantle (or outer core). The solid outer crust is understood as the region of the star spanning about 7 orders of magnitude in density; from about $10^{4} \mathrm{~g} / \mathrm{cm}^{3}$ to $4 \times 10^{11} \mathrm{~g} / \mathrm{cm}^{3}$ [1]. At these densities, the electrons - which are an essential component of the star in order to maintain charge neutrality - have been pressure ionized and move freely throughout the crust. In contrast, at these extremely low nuclear densities the uniform state is unstable against cluster formation. Hence, at the lowest densities of the outer crust, the nucleons cluster into ${ }^{56} \mathrm{Fe}$ nuclei which in turn arrange themselves in a crystalline face-centered-cubic lattice in order to minimize their overall Coulomb repulsion. However, as the density increases (as one moves deeper into the star) ${ }^{56} \mathrm{Fe}$ - with the smallest mass per baryon - is no longer the most energetically favorable nucleus. This is because the electronic contribution to the energy increases faster with density than the corresponding nuclear contribution [1, 2]. As a result, it becomes energetically advantageous for the energetic electrons to capture on the protons and for the excess energy to be carried away by neutrinos. The resulting nuclear lattice is now made of nuclei having a neutron excess larger than that of ${ }^{56} \mathrm{Fe}$. As the density continues to increase, the nuclear system evolves into a Coulomb lattice of progressively more neutron-rich nuclei until a "critical" density of about $4 \times 10^{11} \mathrm{~g} / \mathrm{cm}^{3}$ is reached. At this point the nuclei are unable to hold any more neutrons: the neutron drip line has been reached. Thus, the physics of the outer crust is dominated by an interesting competition between an electronic contribution that attempts to drive the system toward more neutron rich nuclei (i.e., smaller proton fractions) and a nuclear symmetry energy that opposes such a change [2]. Clearly, both the sequence of neutron-rich nuclei as well as the neutron-drip density depend critically on the symmetry energy, which imposes a penalty on the system as it departs from the symmetric $(N=Z=A / 2)$ limit. However, nuclear masses with large neutron excess in the region of the outer crust $(Z \simeq 26-50)$ are presently unknown. Thus, state-of-the-art facilities for rare isotope beams (FRIB) that will map the limits of nuclear existence - such as the one recently commissioned at Michigan State University — will be instrumental in constraining the structure and composition of the outer crust.

\section{B. The Inner Crust}

The inner crust of the neutron star comprises the region from neutron-drip density $(\approx$ $4 \times 10^{11} \mathrm{~g} / \mathrm{cm}^{3}$ ) up to the density at which uniformity in the system is restored (approximately $1 / 3$ to $1 / 2$ of normal nuclear matter saturation density). At these densities the system exhibits rich and complex structures that emerge from a dynamical competition between short-range nuclear attraction and long-range Coulomb repulsion. At the lower densities present in the outer crust, these length scales are well separated and the system organizes itself into a crystalline lattice of neutron-rich nuclei, while at densities of the order of half nuclear-matter saturation density, uniformity in the system is restored. Yet the transition region from the highly-ordered crystal to the uniform liquid mantle is complex and not well understood. Length scales that were well separated in both the crystalline and uniform phases are now comparable, giving rise to "Coulomb frustration". It has been speculated that the transition to the uniform phase must go through a series of changes in the dimensionality and topology of these complex structures - known as "nuclear pasta" [3, 4].

In Fig. 2 a snapshot obtained from a Monte-Carlo simulation of a nuclear system at 


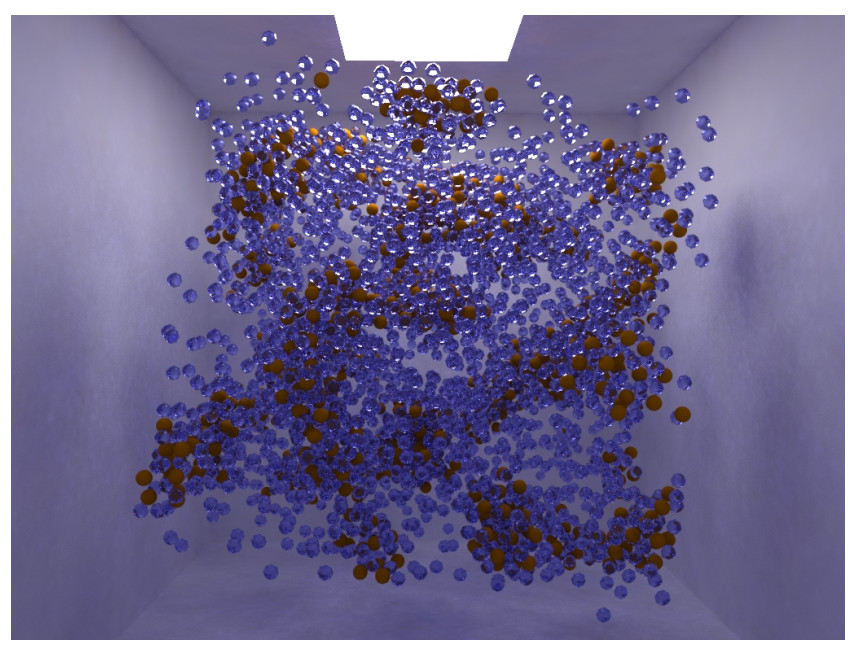

FIG. 2: A snapshot of a Monte Carlo simulation for a configuration of 4,000 nucleons at a baryon density of $0.025 \mathrm{fm}^{-3}$ (a sixth of normal nuclear matter saturation density), a proton fraction of $Z / A=0.2$, and a temperature of $1 \mathrm{MeV}$.

densities relevant to the inner crust is displayed [5, 6]. The figure displays how the system organizes itself into neutron-rich clusters (i.e., "nuclei") of complex topologies that are surrounded by a vapor of (perhaps superfluid) neutrons. Such complex pasta structures may have a significant impact on the propagation of neutrinos and electrons throughout the star.

Interestingly enough, a seemingly unrelated condensed-matter problem - that of the strongly-correlated electron gas - appears to have a very close connection to the exotic pasta phases. In the case of the electron gas, one is interested in understanding the transition between the low-density Wigner crystal and the uniform high-density Fermi liquid. It has been argued that where a first order phase transition is expected, intermediate "microemulsions" (i.e., pasta-like) phases with certain universal characteristics occur instead. Indeed, in two-spatial dimensions a new theorem implies that first-order phase transitions are forbidden in the presence of long-range (e.g., Coulomb) forces[7]. Note that no generalization of this theorem exists in three dimensions. Hence, although the existence of pasta phases in neutron stars may be plausible [3, 4], Oyamatsu and Iida have shown in a recent work that pasta formation may not be universal [8]. Rather, the formation (or lack-thereof) of pasta phases is intimately related to the density dependence of the symmetry energy, a quantity that is poorly known. In particular, the authors conclude that pasta formation requires models with a stiff symmetry energy. Although at present poorly known, the density dependence of the symmetry energy may be accurately determined at the Jefferson Laboratory. The Parity Radius Experiment (PREx) promises to measure the skin thickness of ${ }^{208} \mathrm{~Pb}$ accurately and model independently via parity-violating electron scattering [9, 10]. PREx will provide a unique experimental constraint on the density dependence of the symmetry energy due its strong correlation to the neutron skin of heavy nuclei [11.

\section{The Core}

Whereas the non-uniform crust displays fascinating and intriguing dynamics, its structural impact on the star is rather modest. Indeed, the crust — with a thickness of approximately $1 \mathrm{~km}$ - represents $10 \%$ of the size of the neutron star and contains only about 
$2 \%$ of its mass. The range of densities span by the stellar core go from about $1 / 2$ up to several times nuclear matter saturation density. The highest density attained in the core depends critically on the equation of state of neutron-rich matter which at those high densities is poorly constrained. The cleanest constraint on the equation of state at high-density will emerge as we answer one of the central questions in nuclear astrophysics: what is the maximum mass of a neutron star? Or equivalently, what is the minimum mass of a black hole?

During the past few years some enlightening correlations between finite nuclei and several neutron-star properties have been established [12, 13, 14, 15]. This may at first appear surprising as a heavy nucleus (such as ${ }^{208} \mathrm{~Pb}$ ) is 18 order of magnitudes smaller and 55 orders of magnitude lighter than a neutron star. Yet remarkably, both the neutron radius of a heavy nucleus and the radius of a neutron star depend critically on the pressure of neutron-rich matter. Such correlation among objects of such a disparate size is not difficult to understand. Heavy nuclei develop a neutron-rich skin as a result of both a large neutron excess and a Coulomb barrier that reduces the proton density at the surface of the nucleus. The neutron skin depends critically on the pressure (which is related to the derivative of the energy with respect to the density) exerted on the surface neutrons. It is precisely this same pressure that supports a neutron star against gravitational collapse. Thus models that predict larger neutron skins in heavy nuclei also tend to predict larger neutron star radii. This correlation is displayed in Fig. 4. In this figure two families of accurately calibrated relativistic mean field models (NL3 [16, 17] and "FSUGold" [18]) are employed to generate a range of values for the poorly known neutron skin in ${ }^{208} \mathrm{~Pb}$. It is observed that the stiffer the equation of state of neutron-rich matter, the larger the neutron skin of ${ }^{208} \mathrm{~Pb}$ [11] and the larger the corresponding neutron-star radius. Moreover, the figure includes a hypothetical point that may be determined once both the neutron radius of a heavy nucleus and the neutron radius and mass of neutron star are accurately determined. If such a determination reveals a large neutron skin in ${ }^{208} \mathrm{~Pb}$ (suggesting a stiff EOS at low densities) but a small neutron-star radius (suggesting a "softening" of the EOS at high densities) this may be indicative of a phase transition to an exotic state of matter, such as meson condensates or quark matter.

\section{FORMALISM}

To establish the fundamental role played by the equation of state on the structure of neutron stars (indeed, on any compact object in hydrostatic equilibrium) we introduce the Tolman-Oppenheimer-Volkoff ( $T O V$ ) equations, which represent the extension of Newton's laws to the domain of general relativity. The TOV equations may be expressed as a coupled set of first-order differential equations of the following form:

$$
\begin{aligned}
& \frac{d P}{d r}=-G \frac{\mathcal{E}(r) M(r)}{r^{2}}\left[1+\frac{P(r)}{\mathcal{E}(r)}\right]\left[1+\frac{4 \pi r^{3} P(r)}{M(r)}\right]\left[1-\frac{2 G M(r)}{r}\right]^{-1}, \\
& \frac{d M}{d r}=4 \pi r^{2} \mathcal{E}(r)
\end{aligned}
$$

where $G$ is Newton's gravitational constant, while $P(r), \mathcal{E}(r)$, and $M(r)$ represent the pressure, energy density, and enclosed-mass profiles of the star, respectively. The last three terms (enclosed in square brackets) in Eq. (1a) represent the extension of Newton's laws to the 


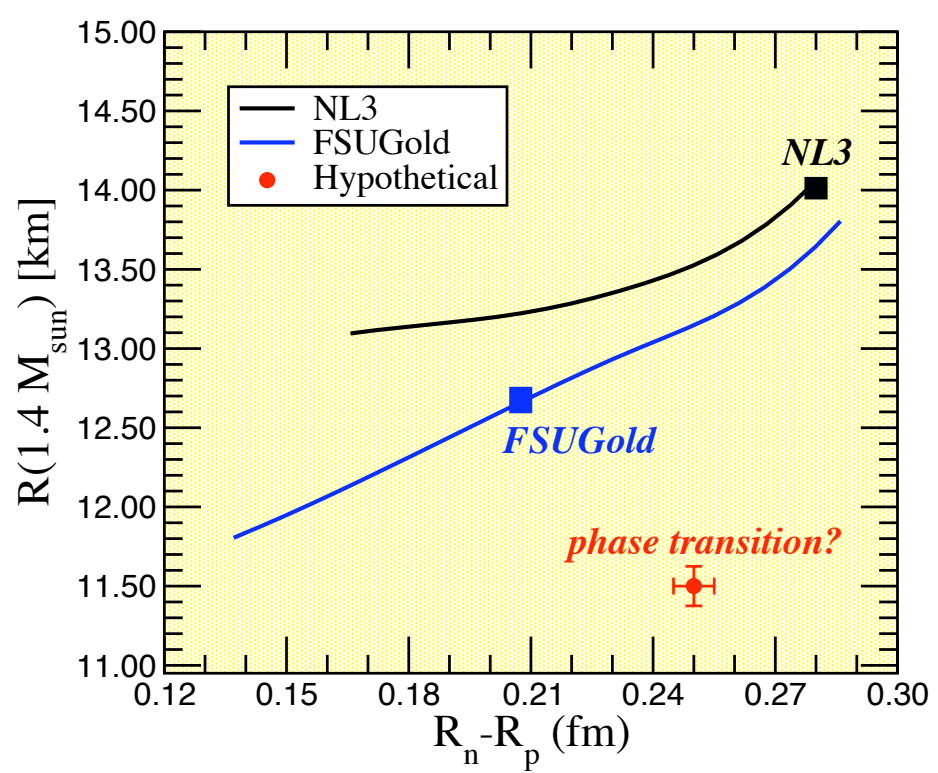

FIG. 3: The radius of a 1.4 solar-mass neutron star as a function of the neutron skin in ${ }^{208} \mathrm{~Pb}$ for two families of accurately calibrated relativistic mean field models (NL3 and FSUGold). Note that models that predict larger neutron skins tend to also predict larger neutron-star radii.

relativistic domain. To the extent that neutron stars may be regarded as spherically symmetric objects in hydrostatic equilibrium, the use of the TOV equations hinges exclusively on the validity of general relativity, an extremely safe assumption. Remarkably then, the only input that neutron stars are sensitive to is the equation of state of neutron-rich matter. This may be easily appreciated as the changes in pressure and enclosed mass as a function of radius (left-hand side of the equations) depend not only on the values of these quantities at $r$, but also on the "unknown" energy density $\mathcal{E}(r)$ of the system. Thus, no solution of the TOV equations is possible until an equation of state (namely, a $P$ vs $\mathcal{E}$ relation) is supplied.

Relativistic mean-field descriptions of the ground-state properties of medium to heavy nuclei have enjoyed enormous success. These highly economical descriptions encode a great amount of physics in a handful of model parameters that are calibrated to a few ground-state properties of a representative set of medium to heavy nuclei. An example of such a successful paradigm is the relativistic NL3 parameter set of Lalazissis, Ring, and collaborators [16, 17].

The Lagrangian density employed in this work is rooted on the seminal work of Walecka, Serot, and their many collaborators (see Refs. [19, 20, 21] and references therein). Since first published by Walecka more than three decades ago [19], several refinements have been implemented to improve the quantitative standing of the model. In the present work we employ an interacting Lagrangian density of the following form [12, 18, 22]:

$$
\begin{aligned}
\mathscr{L}_{\text {int }} & =\bar{\psi}\left[g_{\mathrm{s}} \phi-\left(g_{\mathrm{v}} V_{\mu}+\frac{g_{\rho}}{2} \tau \cdot \mathbf{b}_{\mu}+\frac{e}{2}\left(1+\tau_{3}\right) A_{\mu}\right) \gamma^{\mu}\right] \psi \\
& -\frac{\kappa}{3 !}\left(g_{\mathrm{s}} \phi\right)^{3}-\frac{\lambda}{4 !}\left(g_{\mathrm{s}} \phi\right)^{4}+\frac{\zeta}{4 !}\left(g_{\mathrm{v}}^{2} V_{\mu} V^{\mu}\right)^{2}+\Lambda_{\mathrm{v}}\left(g_{\rho}^{2} \mathbf{b}_{\mu} \cdot \mathbf{b}^{\mu}\right)\left(g_{\mathrm{v}}^{2} V_{\mu} V^{\mu}\right) .
\end{aligned}
$$

The original Lagrangian density of Walecka consisted of an isodoublet nucleon field $(\psi)$ together with neutral scalar $(\phi)$ and vector $\left(V^{\mu}\right)$ fields coupled to the scalar density $(\bar{\psi} \psi)$ and conserved nucleon current $\left(\bar{\psi} \gamma^{\mu} \psi\right)$, respectively [19]. In spite of its simplicity (indeed, the model contains only two dimensionless coupling constants), symmetric nuclear matter 
saturates even when the model was solved at the mean-field level [19]. By adding additional contributions from a single isovector meson $\left(\mathbf{b}^{\mu}\right)$ and the photon $\left(A^{\mu}\right)$, Horowitz and Serot [23] obtained results for the ground-state properties of finite nuclei that rivaled some of the most sophisticated non-relativistic calculations of the time. However, whereas the two dimensionless parameters in the original Walecka model could be adjusted to reproduce the nuclear saturation point, the incompressibility coefficient (now a prediction of the model) came out too large $(K>500 \mathrm{MeV})$ as compared with existing data on breathing-mode energies [24]. To overcome this problem, Boguta and Bodmer introduced cubic $(\kappa)$ and quartic $(\lambda)$ scalar meson self-interactions that accounted for a significant softening of the equation of state $(K=150 \pm 50 \mathrm{MeV})[25]$.

\section{RESULTS}

The two parameters of the Lagrangian density that remain to be discussed are $\zeta$ and $\Lambda_{\mathrm{v}}$. To underscore their fundamental role we now proceed to compute the equation of state of nuclear matter (namely, the energy per particle as a function of density and neutron excess) and its impact on the Mass vs Radius relationship of neutron stars. To capture the behavior of the EOS with neutron excess we display in Fig. 4 the EOS for the two extreme cases of symmetric-nuclear and pure-neutron matter. From the EOS the various bulk parameters that characterize the behavior of neutron-rich matter around saturation density may be extracted; these parameters are listed in Table I1. For a more detailed discussion of the evolution of the EOS with neutron excess, including a precise definition of the various bulk parameters, see Ref. [26]. Note that the symmetry energy is given to a very good approximation as the difference between the energy-per-particle of pure neutron matter and that of symmetric nuclear matter. As we have done elsewhere [27], our results were generated using two accurately calibrated models: NL3 [16, 17] and FSUGold [18].
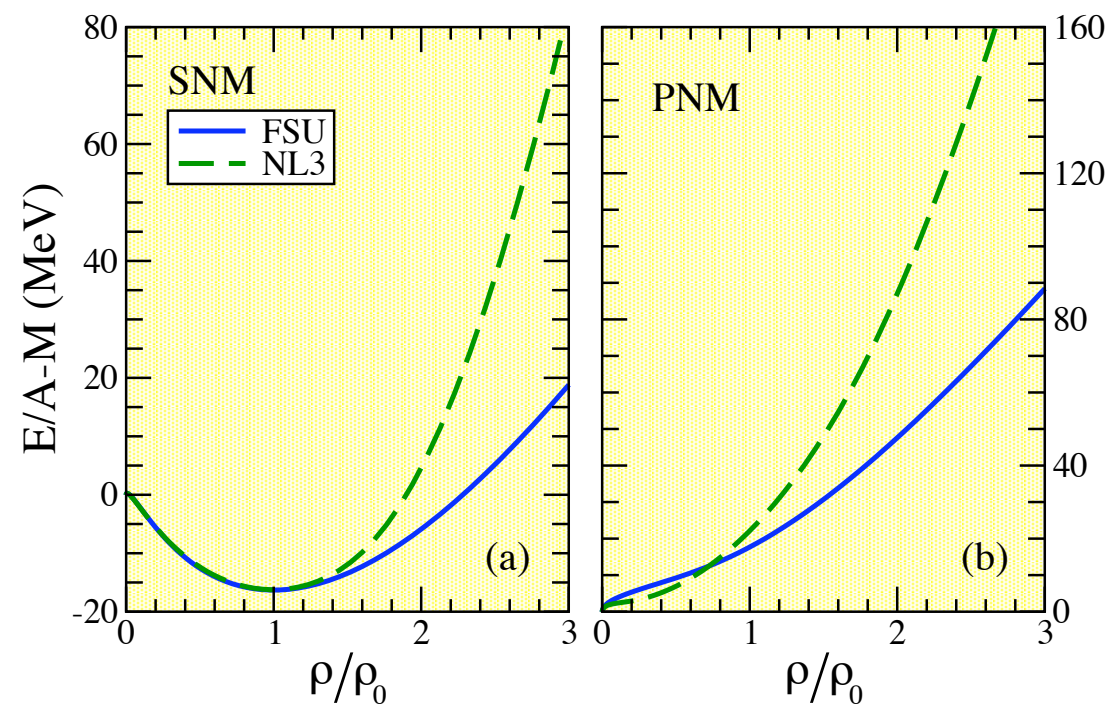

FIG. 4: Equation of state (energy per particle as a function of baryon density) for symmetric matter (a) and pure neutron matter (b) as predicted by the FSUGold (blue solid line) and NL3 (green dashed line) models. 


\begin{tabular}{|l||c|c|c|c|c|c|c|c|}
\hline Model & $\rho_{0}$ & $\varepsilon_{0}$ & $K_{0}$ & $Q_{0}$ & $J$ & $L$ & $K_{\text {sym }}$ & $\gamma$ \\
\hline FSU & 0.148 & -16.30 & 230.0 & -523.4 & 32.59 & 60.5 & -51.3 & 0.64 \\
NL3 & 0.148 & -16.24 & 271.5 & +204.2 & 37.29 & 118.2 & +100.9 & 0.98 \\
\hline
\end{tabular}

TABLE I: Bulk parameters characterizing the energy of symmetric nuclear matter and the symmetry energy at saturation density. All quantities are in $\mathrm{MeV}$, with the exception of $\rho_{0}$ given in $\mathrm{fm}^{-3}$ and the dimensionless parameter $\gamma$. For a detailed explanation of all these quantities see Ref. [26].

Whereas the FSUGold and NL3 models agree on the energy and density at saturation, quantities that are tightly constrained by existent ground-state observables, significant discrepancies emerge in all remaining parameters. The main difference between the two models may be succinctly summarized by stating that whereas FSUGold predicts a soft behavior for both symmetric nuclear matter (through $K_{0}$ ) and the symmetry energy (through $L$ ), NL3 predicts a stiff behavior for both. Note that "stiff" or "soft" refers to whether the energy increases rapidly or slowly with density.

It is worth noting that in the enormously successful NL3 model both parameters $\zeta$ and $\Lambda_{\mathrm{v}}$ are set to zero, suggesting that the experimental data used in the calibration procedure is insensitive to the physics encoded in these parameters. Indeed, Müller and Serot found possible to build models with different values of $\zeta$ that reproduce the same observed properties at normal nuclear densities, but which yield maximum neutron star masses that differ by almost one solar mass [22]. This result indicates that observations of massive neutron stars - rather than laboratory experiments - may provide the only meaningful constraint on the high-density component of the equation of state. Finally, the isoscalar-isovector coupling constant $\Lambda_{\mathrm{v}}$ was added in Ref. [12] to soften the density dependence of the symmetry energy, which is found to be traditionally hard in relativistic mean-field models. Note that this softening was accomplished without compromising the success of the model in reproducing ground-state observables that are accurately measured, such as nuclear masses and charge radii. The introduction of $\Lambda_{\mathrm{v}}$ yields a significant softening of the density dependence of the symmetry energy and, correspondingly, significant reductions in both the neutron skin of heavy nuclei and the radii of neutron stars [13. To illustrate this behavior we display in Fig. 5 the Mass vs Radius relationship of a neutron star. The curves are labeled by their corresponding neutron-skin thickness in ${ }^{208} \mathrm{~Pb}$. Note that the reduction in the value predicted by FSUGold relative to NL3 is due to the inclusion of $\Lambda_{\mathrm{v}}$; so is the reduction in neutron-star radii. For example, for a 1.4 solar-mass neutron star the reduction in the neutron-star radius is close to $2 \mathrm{~km}$. As previously asserted, this correlation suggests how the measurement of the neutron radius of a single heavy nucleus - as will be done at the Jefferson Laboratory in the case of ${ }^{208} \mathrm{~Pb}$ - will constrain the radii of neutron stars. In contrast, the reduction in the maximum neutron-star mass that can be supported by the EOS is controlled by the non-linear vector coupling $\zeta$. This parameter is at present (and perhaps forever) unconstrained by laboratory experiments and its determination awaits the identification of massive neutron stars.

\section{CONCLUSIONS}

The fundamental role played by the equation of state of cold neutron-rich matter in determining the properties of neutron stars was emphasized. Indeed, we observed how 


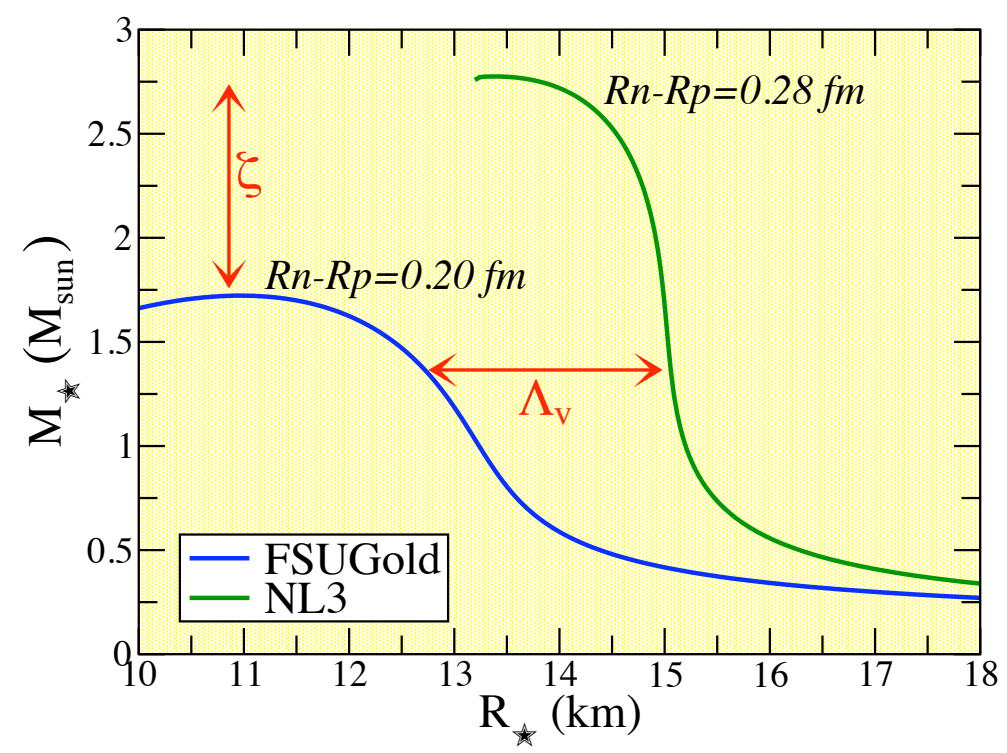

FIG. 5: Predictions for the Mass vs Radius relationship of a neutron star according to the FSUGold (blue solid line) and NL3 (green dashed line) models.

the properties of spherical neutron stars in hydrostatic equilibrium are only sensitive to the equation of state. As such, neutron stars provide a unique window into the behavior of nuclear systems under extreme conditions of density and neutron excess. In this contribution I underscored the special role that nuclear physics plays in the determination of the various fascinating phases displayed in neutron stars and the important role that a concerted effort by theory, experiment, and observation will play in their elucidation.

\section{Acknowledgments}

The author wishes to thank his many collaborators that were involved in this work. This work was supported in part by United States Department of Energy under grant DE-FD0592ER40750. 
[1] G. Baym, C. Pethick, and P. Sutherland, Astrophys. J. 170, 299 (1971).

[2] X. Roca-Maza and J. Piekarewicz, Phys. Rev. C78, 025807 (2008), 0805.2553.

[3] D. G. Ravenhall, C. J. Pethick, and J. R. Wilson, Phys. Rev. Lett. 50, 2066 (1983).

[4] M. Hashimoto, H. Seki, and M. Yamada, Prog. Theor. Phys. 71, 320 (1984).

[5] C. J. Horowitz, M. A. Perez-Garcia, and J. Piekarewicz, Phys. Rev. C69, 045804 (2004), astro-ph/0401079.

[6] C. J. Horowitz, M. A. Perez-Garcia, J. Carriere, D. K. Berry, and J. Piekarewicz, Phys. Rev. C70, 065806 (2004), astro-ph/0409296.

[7] R. Jamei, S. Kivelson, and B. Spivak, Physical Review Letters 94, 056805 (2005).

[8] K. Oyamatsu and K. Iida, Phys. Rev. C75, 015801 (2007), nucl-th/0609040.

[9] C. J. Horowitz, S. J. Pollock, P. A. Souder, and R. Michaels, Phys. Rev. C63, 025501 (2001), nucl-th/9912038.

[10] R. Michaels, P. A. Souder, and G. M. Urciuoli (2005), URL http://hallaweb.jlab.org/ parity/prex.

[11] B. A. Brown, Phys. Rev. Lett. 85, 5296 (2000).

[12] C. J. Horowitz and J. Piekarewicz, Phys. Rev. Lett. 86, 5647 (2001), astro-ph/0010227.

[13] C. J. Horowitz and J. Piekarewicz, Phys. Rev. C64, 062802 (2001), nucl-th/0108036.

[14] C. J. Horowitz and J. Piekarewicz, Phys. Rev. C66, 055803 (2002), nucl-th/0207067.

[15] A. W. Steiner, M. Prakash, J. M. Lattimer, and P. J. Ellis, Phys. Rept. 411, 325 (2005), nucl-th/0410066.

[16] G. A. Lalazissis, J. Konig, and P. Ring, Phys. Rev. C55, 540 (1997), nucl-th/9607039.

[17] G. A. Lalazissis, S. Raman, and P. Ring, At. Data Nucl. Data Tables 71, 1 (1999).

[18] B. G. Todd-Rutel and J. Piekarewicz, Phys. Rev. Lett 95, 122501 (2005), nucl-th/0504034.

[19] J. D. Walecka, Annals Phys 83, 491 (1974).

[20] B. D. Serot and J. D. Walecka, Adv. Nucl. Phys. 16, 1 (1986).

[21] B. D. Serot and J. D. Walecka, Int. J. Mod. Phys. E6, 515 (1997), nucl-th/9701058.

[22] H. Mueller and B. D. Serot, Nucl. Phys. A606, 508 (1996), nucl-th/9603037.

[23] C. J. Horowitz and B. D. Serot, Nucl. Phys. A368, 503 (1981).

[24] D. H. Youngblood, C. M. Rozsa, J. M. Moss, D. R. Brown, and J. D. Bronson, Phys. Rev. Lett. 39, 1188 (1977).

[25] J. Boguta and A. R. Bodmer, Nucl. Phys. A292, 413 (1977).

[26] J. Piekarewicz and M. Centelles (2008), 0812.4499.

[27] J. Piekarewicz, Phys. Rev. C76, 031301 (2007), 0705.1491. 